\title{
An Inventory Model for Perishable Items with Time Varying Stock Dependent Demand and Trade Credit under Inflation
}

\author{
Sushil Kumar*, U. S. Rajput \\ Department of Mathematics \& Astronomy, University of Lucknow, Lucknow, India \\ Email: ${ }^{*}$ sushilmath4444@gmail.com
}

Received 21 August 2015; accepted 20 September 2015; published 23 September 2015

Copyright (C) 2015 by authors and Scientific Research Publishing Inc.

This work is licensed under the Creative Commons Attribution International License (CC BY). http://creativecommons.org/licenses/by/4.0/

c) (i) Open Access

\begin{abstract}
In the classical inventory models, it is assumed that the retailer pays to the supplier as soon as he received the items and in such cases the supplier offers a cash discount or credit period (permissible delay) to the retailer. In this paper we presented an inventory model for perishable items with time varying stock dependent demand under inflation. It is assumed that the supplier offers a credit period to the retailer and the length of credit period is dependent on the order quantity. The purpose of our study is to minimize the present value of retailer's total cost. Numerical examples are also given to demonstrate the presented mode.
\end{abstract}

\section{Keywords}

Inventory, Deterioration, Perishable, Credit Period, Time Varying Stock Dependent Demand

\section{Introduction}

In the classical inventory models payment for the items paid by the supplier depends on the payment paid by the retailer and in such cases the supplier offers a fixed credit period to the retailer during which no interest will be charged by the supplier so there is no need to pay the purchasing cost by the retailer and after this credit period up to the end of a period interest charged and paid by the retailer. In such situations the retailer starts to accumulate revenue on his sale and earn interest on his revenue. If the revenue earned by the retailer up to the end of credit period is enough to pay the purchasing cost or there is a budget then the balance is settled and the supplier does not charge any interest, otherwise the supplier charges interest for unpaid balance after the credit period. The interest and the remaining payment are made at the end of replenishment cycle.

\footnotetext{
${ }^{*}$ Corresponding author.
} 
In traditional EOQ models the payment time does not affect the profit and replenishment policy. If we consider the inflation then order quantity and payment time can influence both the supplier's and retailer's decisions. A large pile of perishable foods such as fruits, vegetables, milk, bread, chocklet etc. attract the consumers to buy more. Buzacott [1] considered an EOQ model with different type of pricing policies under inflation. Silver and Peterson [2] developed an inventory model and show that the consumption rate is proportional to the displayed stock level. Baker and Urban [3] proposed an inventory model for deteriorating items with the demand rate is a polynomial function of instantaneous stock level. Mandal and Phaujdar [4] presented an inventory model for deteriorating items with stock level dependent consumption rate. Vrat and Padmanabhan [5] considered an inventory model with stock dependent demand under constant inflation rate. Padmanabhan and Vrat [6] developed an EOQ model for perishable products under stock dependent selling rate. Bose et al. [7] considered an inventory model for deteriorating items with time dependent demand and shortages under inflation and time discounting. Mandal and Maiti [8] developed an inventory model for damageable items with stock dependent demand and variable replenishment rate. Chung and Lin [9] determine an optimal replenishment policy for an inventory model of deteriorating items by considering inflation and credit period. Chang [10] proposed an EOQ model for deteriorating items under inflation and time discounting assuming that the supplier offers a trade credit policy if the retailer order size is larger than a certain level. Dye and Ouyang [11] developed an EOQ model for perishable items with stock dependent selling rate by allowing shortages. Hou [12] presented an inventory model for deteriorating items with stock dependent consumption rate and shortages under inflation and credit period. Jaggi et al. [13] determine an optimal ordering policy for deteriorating items under inflation induced demand. Sana and Chaudhuri [14] developed a deterministic EOQ model with stock dependent demand and delay in payments. Valliaththal and Uthayakumar [15] presented an inventory model for perishable items under stock and time dependent selling rate with shortages. Roy et al. [16] considered an inventory model for deteriorating items with stock dependent demand under fuzzy inflation and time discounting over a random planning horizon. Sana [17] proposed a lot size inventory model with stock dependent demand and time varying deterioration and partial backlogging. Chang et al. [18] determine an optimal replenishment policy for an inventory model of non-instantaneous deteriorating items with stock dependent demand. Sarkar et al. [19] presented an EMQ (economic manufacturing quantity) model of an imperfect production process with time dependent demand and time value of money under inflation. Yan [20] considered an EOQ model for perishable items with freshness dependent demand and partial backlogging. Nagrare and Dutta [21] developed a continuous review inventory model for perishable products with inventory dependent demand. Sana [22] proposed a control policy for a production system inflation assuming a stock dependent demand and sales team promotional effort.Shuai et al. [23] considered an inventory model for perishable products with stock dependent demand and trade credit under inflation.

Table 1 and Table 2 show the variation of the parameters $\mathrm{r}$ and $\mathrm{M}$ when $M \geq T$ and Table 3 \& Table 4 show the variation of parameters $r$ and $M$ when $M \prec T$. Figure $1 \&$ Figure 2 are correspond to the developed model. Figure 3 \& Figure 4 show the variation of retailer's total cost with respect to the parameters $r$ and $M$ when $M \geq T$ and Figure 5 \& Figure 6 show the variation of retailer's total cost with respect to the parameters $\mathrm{r}$ and $\mathrm{M}$ when $M \prec T$.

In the present paper we presented an inventory model for perishable items with time varying stock dependent demand and trade credit under inflation. Although there are so many research papers related to the perishable

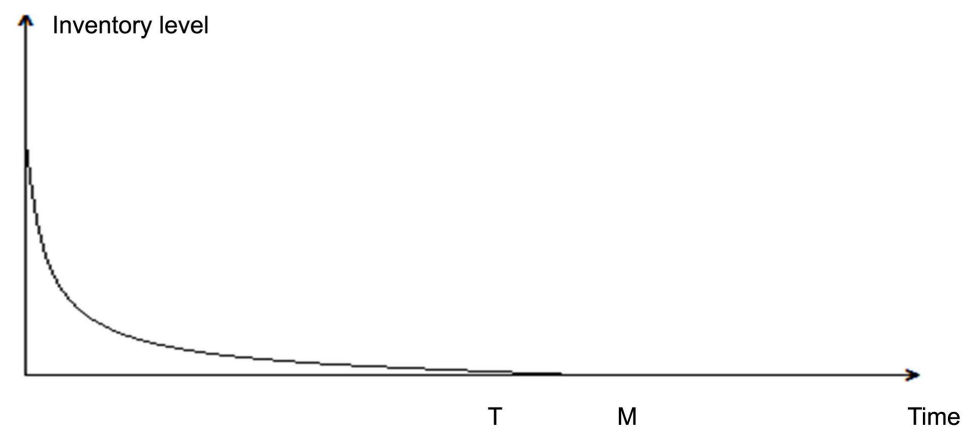

Figure 1. Corresponding to developed model. 


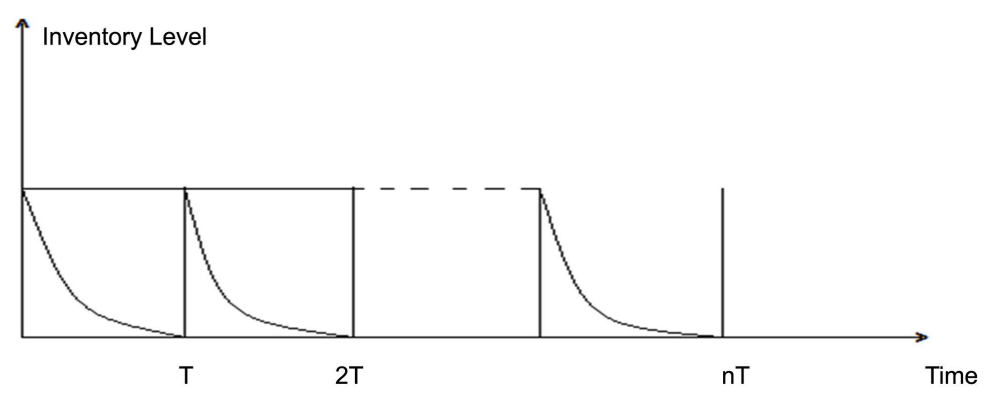

Figure 2. With $n$ cycles in the developed model.

Table 1. Variation of retailer's total cost with respect to the change of parameter $r$.

\begin{tabular}{ccc}
\hline$r$ & $T$ & $T_{1}(T)$ \\
1.2 & 0.809153 & $-3.18984 \times 10^{6}$ \\
1.4 & 1.29391 & $-7.38628 \times 10^{6}$ \\
1.6 & 1.70349 & $-1.360695 \times 10^{7}$ \\
1.8 & 2.07928 & $-2.238335 \times 10^{7}$ \\
2 & 2.43731 & $-3.4213250 \times 10^{7}$ \\
2.2 & 2.78705 & $-4.963000 \times 10^{7}$ \\
2.5 & 3.30941 & $-8.063075 \times 10^{7}$ \\
\hline
\end{tabular}

Table 2. Variation of retailer's total cost with respect to the change of parameter $M$.

\begin{tabular}{ccc}
\hline$M$ & $T$ & $T_{1}(T)$ \\
\hline 2 & 0.809153 & $-3.18984 \times 10^{6}$ \\
4 & 3.71242 & $-6.82474 \times 10^{7}$ \\
6 & 6.66987 & $-2.96128 \times 10^{8}$ \\
8 & 9.64061 & $-7.88637 \times 10^{8}$ \\
10 & 12.6158 & $-1.64743 \times 10^{9}$ \\
12 & 15.5929 & $-2.97412 \times 10^{9}$ \\
\hline
\end{tabular}

Table 3. Variation of retailer's total cost with respect to the change of parameter $r$.

\begin{tabular}{cccc}
\hline$r$ & $T$ & $T C H_{2}(T)$ & $-4.59988 \times 10^{7}$ \\
0.05 & 0.494393 & $-4.30252 \times 10^{7}$ \\
0.1 & 0.500148 & $-4.02547 \times 10^{7}$ \\
0.15 & 0.500591 & $-3.78662 \times 10^{7}$ \\
0.2 & 0.495351 & $-3.58238 \times 10^{7}$ \\
0.25 & 0.483842 & $-3.41820 \times 10^{7}$ \\
0.35 & 0.465167 & 0.437937 & $-3.30523 \times 10^{7}$ \\
0.4 & 0.399872 & $-3.26667 \times 10^{7}$ \\
\hline
\end{tabular}


Table 4. Variation of retailer's total cost with respect to the change of parameter $M$.

\begin{tabular}{ccc}
\hline$M$ & $T$ & $T_{2}(T)$ \\
\hline 2 & 0.494393 & $-4.59988 \times 10^{7}$ \\
4 & 1.02028 & $-3.86733 \times 10^{8}$ \\
6 & 1.54603 & $-1.32099 \times 10^{9}$ \\
8 & 2.07174 & $-3.13999 \times 10^{9}$ \\
10 & 2.59744 & $-6.03636 \times 10^{9}$ \\
12 & 3.12314 & $-1.05591 \times 10^{10}$ \\
\hline
\end{tabular}

\section{TC}

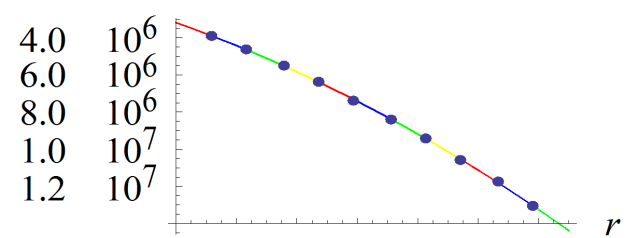

$\begin{array}{llllll}1.4 & 1.6 & 1.8 & 2.0 & 2.2 & 2.4\end{array}$

Figure 3. Variation in TC with respect to $r$.

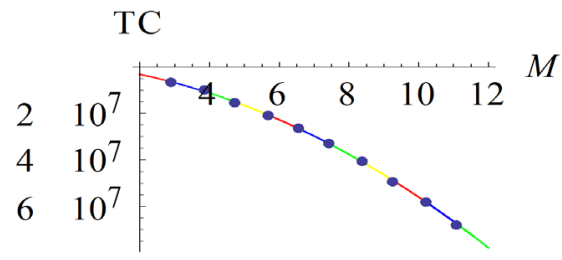

Figure 4. Variation in TC with respect to $M$.

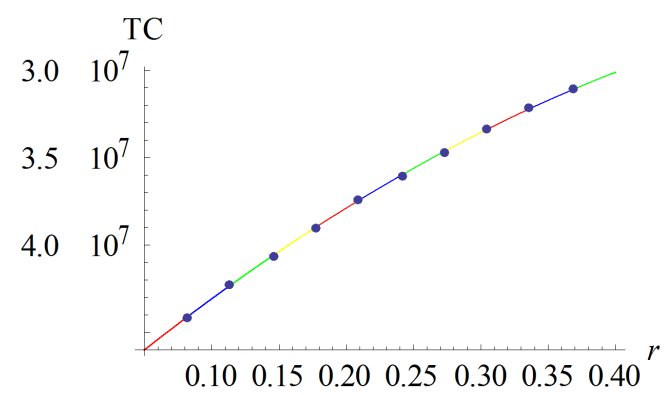

Figure 5. Variation in TC with respect to $r$.

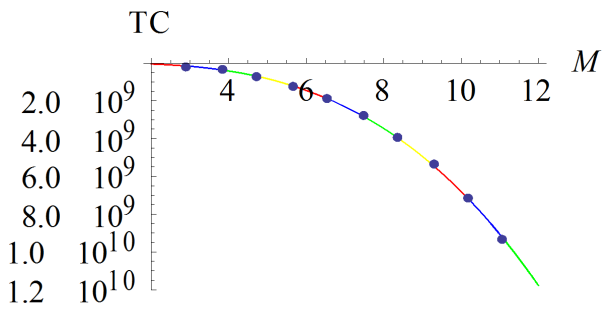

Figure 6. Variation in TC with respect to $M$. 
products with stock dependent demand under inflation. This paper deals with the same type problem and it provides an approximate solution procedure of this problem for minimizing the present value of retailer's total cost.

\section{Assumptions and Notations}

We consider the following assumptions and notations corresponding to the developed model

1) The demand rate $R(t)$ is $R(t)=a+b t+k I(t), a \geq 0,0 \leq b \leq 1, k \succ 0$

2) $\theta$ is the constant deterioration rate.

3) $o_{C}$ is the ordering cost per order.

4) $h_{C}$ is the holding cost per unit.

5) $s_{C}$ is the shortage cost.

6) $M$ is the credit period.

7) $T$ is the replenish cycle length.

8) $r$ is the inflation rate.

9) $I_{C}$ is the interest charged per \$ per unit time when $T \succ M$.

10) $C$ is the purchasing cost per unit.

11) $P$ is the selling price per unit with $P \succ C$.

$12) Q$ is the initial inventory level.

$13) L$ is the planning horizon.

14)The supplier sells one single item to the retailer.

15)The items are replenished when the stock level becomes zero.

16)The supplier provides a credit period, which is dependent on the order quantity.

17)The lead time is zero.

18)Shortages are not allowed.

19)The inventory planning horizon is finite and the numbers of cycles are finite in the planning horizon.

$20) I(t)$ is the inventory level at any time $t$.

\section{Mathematical Formulation}

Suppose an inventory system consists the maximum inventory level at any time $t=0$ and due to both demand and deterioration the inventory level decreases in the interval $[0, T]$. The replenishment cycle starts with the initial maximum inventory level $Q$ and ends with zero stock level. The retailer's instantaneous inventory level at any time $t$ in the interval $[0, T]$ is governed by the following differential equation

$$
\frac{\mathrm{d} I}{\mathrm{~d} t}+\theta I=-[a+b t+k I(t)], \quad 0 \leq t \leq T
$$

With the boundary condition

$$
I(T)=0
$$

The equation (1) can also be written as

$$
\frac{\mathrm{d} I}{\mathrm{~d} t}+\alpha I=-[a+b t], \quad 0 \leq t \leq T
$$

where $\alpha=(\theta+k)$

With the boundary condition

$$
I(T)=0
$$

For a $2^{\text {nd }}$ order approximation of $\mathrm{e}^{-\alpha t}$ and $\mathrm{e}^{\alpha t}$, the solution of Equation (2) is

$$
\begin{aligned}
I= & {\left[\frac{(2 a \alpha+b \alpha-2 b)}{2 \alpha} T-\frac{(2 a \alpha+b \alpha-2 b)}{2 \alpha} t+\frac{(a \alpha+b \alpha-b)}{2} T^{2}+\frac{(a \alpha-b)}{2} t^{2}-\frac{(2 a \alpha+b \alpha-2 b)}{2} T t\right.} \\
& \left.+\frac{\left(2 a \alpha^{2}+b \alpha^{2}-2 b \alpha\right)}{2} t^{3}-\frac{\left(a \alpha^{2}+b \alpha-b \alpha\right)}{2} T^{2} t-\frac{\left(a \alpha^{2}+b \alpha^{2}-b \alpha\right)}{2} T t^{2}\right],
\end{aligned}
$$


Using the boundary condition, $I(0)=Q$ the initial order quantity is

$$
Q=\left[\frac{(2 a \alpha+b \alpha-2 b)}{2 \alpha} T+\frac{(a \alpha+b \alpha-b)}{2} T^{2}\right],
$$

Now we discuss the following two cases

(1) $M \geq T$ and (2) $M \prec T$

\subsection{Case I}

When $M \geq T$ then in this case the retailer can sell all the items before the end of credit period $M$ because the credit period $M$ is greater than the replenishment cycle length so no interest will be charged by the retailer. Since the purchasing cost is paid at the end of credit period $M$.

During the 1 st cycle the present value of ordering cost is

$$
O_{C}=A \text {, }
$$

During the 1st cycle the present value of purchasing cost is

$$
\begin{gathered}
P_{C}=C Q \mathrm{e}^{-r M} \\
P_{C}=\frac{C \mathrm{e}^{-r M}}{2}\left[\frac{(2 a \alpha+b \alpha-2 b)}{2} T+(a \alpha+b \alpha-b) T^{2}\right],
\end{gathered}
$$

During the 1st cycle the present value of holding cost is

$$
\begin{gathered}
H_{C}=h_{C} \int_{0}^{T} I(t) \mathrm{e}^{-r t} \mathrm{~d} t \\
H_{C}=h_{C}\left[\frac{(2 a \alpha+b \alpha-2 b)}{4 \alpha} T^{2}+\frac{(2 a \alpha+3 b \alpha-2 b)}{12} T^{3}-\frac{r(2 a \alpha+b \alpha-2 b)}{12 \alpha} T^{3}\right],
\end{gathered}
$$

Therefore during the 1st cycle the present value of retailer's total cost is

$$
\begin{aligned}
& T C_{1}(T)=\left[O_{C}+H_{C}+P_{C}\right] \\
T C_{1}(T)= & A+\frac{h_{C}}{12 \alpha}\left\{(6 a \alpha+3 b \alpha-6 b) T^{2}+\left(2 a \alpha^{2}+3 b \alpha^{2}-2 b \alpha\right) T^{3}\right. \\
& \left.-r(2 a \alpha+b \alpha-2 b) T^{3}\right\}+\frac{C \mathrm{e}^{-r M}}{2 \alpha}\{(2 a \alpha+b \alpha-2 b) T \\
& \left.\left.+\left(a \alpha^{2}+b \alpha^{2}-b \alpha\right) T^{2}\right\}\right],
\end{aligned}
$$

Since there are m cycles in the planning horizon $L$ then the present value of retailer's total cost over the planning horizon $L$ is

$$
\begin{aligned}
& \operatorname{TCH}_{1}(T)=\sum_{n=0}^{m-1} T C_{1}(T) \mathrm{e}^{-r n T} \\
T C H_{1}(T)= & \frac{\left(1-\mathrm{e}^{-r L}\right)}{\left(1-\mathrm{e}^{-r T}\right)}\left[A+\frac{h_{C}}{12 \alpha}\left\{(6 a \alpha+3 b \alpha-6 b) T^{2}+\left(2 a \alpha^{2}+3 b \alpha^{2}-2 b \alpha\right) T^{3}\right.\right. \\
& \left.-r(2 a \alpha+b \alpha-2 b) T^{3}\right\}+\frac{C \mathrm{e}^{-r M}}{2 \alpha}\{(2 a \alpha+b \alpha-2 b) T \\
& \left.\left.+\left(a \alpha^{2}+b \alpha^{2}-b \alpha\right) T^{2}\right\}\right]
\end{aligned}
$$




$$
\begin{aligned}
T C H_{1}(T)= & L\left[\frac{A}{T}+A r+\frac{A r^{2} T}{4}+\frac{h_{C}}{12 \alpha}\left\{(6 a \alpha+3 b \alpha-6 b) T+\alpha(2 a \alpha+3 b \alpha-2 b) T^{2}\right.\right. \\
& \left.-r(2 a \alpha+b \alpha-2 b) T^{2}\right\}+\frac{C \mathrm{e}^{-r M}}{2 \alpha}\{(2 a \alpha+b \alpha-2 b)+\alpha(a \alpha+b \alpha-b) T\} \\
& +\frac{r h_{C}}{12 \alpha}\left\{(6 a \alpha+3 b \alpha-6 b) T^{2}+\alpha(2 a \alpha+3 b \alpha-2 b) T^{3}-r(2 a \alpha+b \alpha-2 b) T^{3}\right\} \\
& +\frac{r C \mathrm{e}^{-r M}}{2 \alpha}\left\{(2 a \alpha+b \alpha-2 b) T^{2}+\alpha(a \alpha+b \alpha-b) T^{2}\right\} \\
& +\frac{C r^{2} \mathrm{e}^{-r M}}{8 \alpha}\left\{(2 a \alpha+b \alpha-2 b) T^{2}+\alpha(a \alpha+b \alpha-b) T^{3}\right\} \\
& \left.+\frac{r^{2} h_{C}}{48 \alpha}(6 a \alpha+3 b \alpha-6 b) T^{3}\right],
\end{aligned}
$$

The necessary condition for $T C H_{1}(T)$ to be minimum is that $\frac{\mathrm{d} T C H_{1}(T)}{\mathrm{d} T}=0$ and the sufficient condition is $\frac{\mathrm{d}^{2} T C H_{1}(T)}{\mathrm{d} T^{2}} \succ 0$ at the optimum value of $T$.

$$
\begin{aligned}
\frac{\mathrm{d} T C H_{1}(T)}{\mathrm{d} T}= & L\left[\frac{A r^{2}}{4}-\frac{A}{T^{2}}+\frac{h_{C}}{12 \alpha}\{(6 a \alpha+3 b \alpha-6 b)+2 \alpha(2 a \alpha+3 b \alpha-2 b) T\right. \\
& -2 r(2 a \alpha+b \alpha-2 b) T\}+\frac{C \mathrm{e}^{-r M}}{2}(a \alpha+b \alpha-b)+\frac{r h_{C}}{12 \alpha}\{2(6 a \alpha+3 b \alpha-6 b) T \\
& \left.+3 \alpha(2 a \alpha+3 b \alpha-2 b) T^{2}-3 r(2 a \alpha+b \alpha-2 b) T^{2}\right\}+\frac{C r \mathrm{e}^{-r M}}{\alpha}\{(2 a \alpha+b \alpha-2 b) T \\
+ & \alpha(a \alpha+b \alpha-b) T\}+\frac{C r^{2} \mathrm{e}^{-r M}}{8 \alpha}\left\{2(2 a \alpha+b \alpha-2 b)+3 \alpha(a \alpha+b \alpha-b) T^{2}\right\} \\
+ & \left.\frac{r^{2} h_{C}}{16 \alpha}(6 a \alpha+3 b \alpha-6 b) T^{2}\right], \\
\frac{\mathrm{d}^{2} T C H_{1}(T)}{\mathrm{d} T^{2}}= & L\left[\frac{2 A}{T^{3}}+\frac{h_{C}}{6 \alpha}\{\alpha(2 a \alpha+3 b \alpha-2 b)-r(2 a \alpha+b \alpha-2 b)\}\right. \\
& +\frac{r h_{C}}{6 \alpha}\{(6 a \alpha+3 b \alpha-6 b)+3 \alpha(2 a \alpha+3 b \alpha-2 b) T-3 r(2 a \alpha+b \alpha-2 b) T\} \\
& +\frac{C r \mathrm{e}^{-r M}}{\alpha}\{(2 a \alpha+b \alpha-2 b)+\alpha(a \alpha+b \alpha-b)\} \\
& \left.+\frac{3 C r^{2} \mathrm{e}^{-r M}}{4}(a \alpha+b \alpha-b) T+\frac{r^{2} h_{C}}{8 \alpha}(6 a \alpha+3 b \alpha-6 b)\right]
\end{aligned}
$$

\subsection{Case II}

When $M \prec T$ then there are three possibilities

1) Let $P \mathrm{e}^{r M} \int_{0}^{M} R(t) \mathrm{e}^{-r t} \mathrm{~d} t \succ C Q$ then at $M$ the revenue earned by the retailer is more than the purchasing cost so in this case no interest will be charged by the supplier although the credit period $M$ is smaller than the reple- 
nishment cycle length $T$ so the present value of retailer's total will be same as that in case I.

$$
\begin{aligned}
T C H_{1}(T)= & L\left[\frac{A}{T}+A r+\frac{A r^{2} T}{4}+\frac{h_{C}}{12 \alpha}\left\{(6 a \alpha+3 b \alpha-6 b) T+\alpha(2 a \alpha+3 b \alpha-2 b) T^{2}\right.\right. \\
& \left.-r(2 a \alpha+b \alpha-2 b) T^{2}\right\}+\frac{C \mathrm{e}^{-r M}}{2 \alpha}\{(2 a \alpha+b \alpha-2 b)+\alpha(a \alpha+b \alpha-b) T\} \\
& +\frac{r h_{C}}{12 \alpha}\left\{(6 a \alpha+3 b \alpha-6 b) T^{2}+\alpha(2 a \alpha+3 b \alpha-2 b) T^{3}-r(2 a \alpha+b \alpha-2 b) T^{3}\right\} \\
& +\frac{r C \mathrm{e}^{-r M}}{2 \alpha}\left\{(2 a \alpha+b \alpha-2 b) T^{2}+\alpha(a \alpha+b \alpha-b) T^{2}\right\} \\
& +\frac{C r^{2} \mathrm{e}^{-r M}}{8 \alpha}\left\{(2 a \alpha+b \alpha-2 b) T^{2}+\alpha(a \alpha+b \alpha-b) T^{3}\right\} \\
& \left.+\frac{r^{2} h_{C}}{48 \alpha}(6 a \alpha+3 b \alpha-6 b) T^{3}\right],
\end{aligned}
$$

2) Let $P \mathrm{e}^{r M} \int_{0}^{M} R(t) \mathrm{e}^{-r t} \mathrm{~d} t \prec C Q$ then at $M$ the revenue earned by the retailer is less than the purchasing cost and the retailer has a budget to pay the remaining short purchasing cost so in this case there is still no interest charged by the supplier although the credit period $M$ is smaller than the replenishment cycle length $T$ so the present value of retailer's total will be same as that in case I.

$$
\begin{aligned}
T C H_{1}(T)= & L\left[\frac{A}{T}+A r+\frac{A r^{2} T}{4}+\frac{h_{C}}{12 \alpha}\left\{(6 a \alpha+3 b \alpha-6 b) T+\alpha(2 a \alpha+3 b \alpha-2 b) T^{2}\right.\right. \\
& \left.-r(2 a \alpha+b \alpha-2 b) T^{2}\right\}+\frac{C \mathrm{e}^{-r M}}{2 \alpha}\{(2 a \alpha+b \alpha-2 b)+\alpha(a \alpha+b \alpha-b) T\} \\
& +\frac{r h_{C}}{12 \alpha}\left\{(6 a \alpha+3 b \alpha-6 b) T^{2}+\alpha(2 a \alpha+3 b \alpha-2 b) T^{3}-r(2 a \alpha+b \alpha-2 b) T^{3}\right\} \\
& +\frac{r C \mathrm{e}^{-r M}}{2 \alpha}\left\{(2 a \alpha+b \alpha-2 b) T^{2}+\alpha(a \alpha+b \alpha-b) T^{2}\right\} \\
& +\frac{C r^{2} \mathrm{e}^{-r M}}{8 \alpha}\left\{(2 a \alpha+b \alpha-2 b) T^{2}+\alpha(a \alpha+b \alpha-b) T^{3}\right\} \\
& \left.+\frac{r^{2} h_{C}}{48 \alpha}(6 a \alpha+3 b \alpha-6 b) T^{3}\right]
\end{aligned}
$$

3) Let $P \mathrm{e}^{r M} \int_{0}^{M} R(t) \mathrm{e}^{-r t} \mathrm{~d} t \prec C Q$ then at $M$ the revenue earned by the retailer is less than the purchasing cost and the retailer has no budget to pay the remaining short purchasing cost so in this case for unpaid balance the interest will be charged by the supplier from $M$ to $T$. The interest and the remaining payments are made at the end of replenishment cycle. So in this case the retailer's total cost containing the ordering cost, holding cost, purchasing cost paid at $M$, the interest and the remaining payments are made at the end of replenishment cycle.

The present values of retailer's ordering and holding are same cost as in case I

During the first cycle the purchasing cost paid at $M$ is equal to the amount of revenue earned by the retailer up to $M$ so

$$
P_{C}=P \int_{0}^{M} R(t) \mathrm{e}^{-r t} \mathrm{~d} t
$$




$$
\begin{aligned}
P_{C}= & P\left[a M+\frac{b M^{2}}{2}-\frac{a r M^{2}}{2}-\frac{b r M^{3}}{3}+k\left\{\frac{(2 a \alpha+b \alpha-2 b)}{2 \alpha} T M\right.\right. \\
& -\frac{(2 a \alpha+b \alpha-2 b)}{4 \alpha} M^{2}+\frac{(a \alpha+b \alpha-b)}{2} T^{2} M+\frac{(a \alpha-b)}{6} M^{3} \\
& \left.-\frac{(2 a \alpha+b \alpha-2 b)}{4} T M^{2}\right\}-r k\left\{\frac{(2 a \alpha+b \alpha-2 b)}{4 \alpha} T M^{3}\right. \\
& \left.\left.-\frac{(2 a \alpha+b \alpha-2 b)}{6 \alpha} M^{3}+\frac{(a \alpha+b \alpha-b)}{4} T^{2} M^{2}\right\}+\frac{a r^{2} M^{3}}{6}\right],
\end{aligned}
$$

During the first cycle the present values of remaining payments and interest paid at the end of replenishment cycle are

$$
\begin{aligned}
& R P_{I}=\left\{C Q-P \mathrm{e}^{r M} \int_{0}^{M} R(t) \mathrm{e}^{-r t} \mathrm{~d} t\right\}\left[1+I_{C}(T-M)\right] \mathrm{e}^{-r T} \\
& R P_{I}=\left[\frac{C}{2 \alpha}\left(1-I_{C} M\right)(2 a \alpha+b \alpha-2 b) T+\frac{C}{2}\left(1-I_{C} M\right)(a \alpha+b \alpha-b) T^{2}\right. \\
& -P\left(1-I_{C} M\right)\left\{a M+\frac{a r M^{2}}{2}+\frac{b M^{2}}{2}+\frac{b r M^{3}}{6}+\frac{a^{2} r^{2} M^{3}}{6}-\frac{k(2 a \alpha+b \alpha-2 b) M^{2}}{4 \alpha}\right. \\
& \left.+\frac{k(a \alpha-b) M^{3}}{6}-\frac{k r(2 a \alpha+b \alpha-2 b) M^{3}}{12 \alpha}\right\}-\frac{P k\left(1-I_{C} M\right)(2 a \alpha+b \alpha-2 b)}{2 \alpha} T M \\
& -\frac{P k r\left(1-I_{C} M\right)}{2 \alpha}(2 a \alpha+b \alpha-2 b) T M^{2}+\frac{P k(r+1)\left(1-I_{C} M\right)(2 a \alpha+b \alpha-2 b)}{4} T M^{2} \\
& +\frac{P k r\left(1-I_{C} M\right)}{4}(2 a \alpha+b \alpha-2 b) T M^{3}-\frac{P k r\left(1-I_{C} M\right)(a \alpha+b \alpha-b)}{4} T^{2} M^{2} \\
& +\frac{C}{2 \alpha}\left(I_{C}+r M I_{C}-r\right)(2 a \alpha+b \alpha-2 b) T^{2}+\frac{C\left(I_{C}+r M I_{C}-r\right)(a \alpha+b \alpha-b)}{2} T^{3} \\
& -P\left(I_{C}+r M I_{C}-r\right)\left\{a M+\frac{a r M^{2}}{2}+\frac{b M^{2}}{2}+\frac{b r M^{3}}{6}+\frac{a^{2} r^{2} M^{3}}{6}-\frac{k(2 a \alpha+b \alpha-2 b)}{4 \alpha} M^{2}\right. \\
& \left.+\frac{k(a \alpha-b) M^{3}}{6}-\frac{k r(2 a \alpha+b \alpha-2 b) M^{3}}{12 \alpha}\right\} T-\frac{P k\left(I_{C}+r M I_{C}-r\right)}{2 \alpha}(2 a \alpha+b \alpha-2 b) T^{2} M \\
& -\frac{P k r\left(I_{C}+r M I_{C}-r\right)(2 a \alpha+b \alpha-2 b)}{2 \alpha} T^{2} M^{2} \\
& +\frac{P k(r+1)\left(I_{C}+r M I_{C}-r\right)}{4}(2 a \alpha+b \alpha-2 b) T^{2} M^{2} \\
& +\frac{C\left(r^{2}-2 r I_{C}-r^{2} M I_{C}\right)(2 a \alpha+b \alpha-2 b)}{4 \alpha} T^{3} \\
& -\frac{P}{2}\left(r^{2}-2 r I_{C}-r^{2} M I_{C}\right)\left\{a M+\frac{b M^{2}}{2}-\frac{k(2 a \alpha+b \alpha-2 b)}{4 \alpha} M^{2}\right\} T^{2} \\
& \left.-\frac{P k\left(r^{2}-2 r I_{C}\right)(2 a \alpha+b \alpha-2 b)}{4 \alpha} T^{3} M\right],
\end{aligned}
$$

During the 1st cycle the present value of retailer's total cost is 


$$
\begin{aligned}
& T C_{2}(T)=\left[A+\frac{h_{C}}{12 \alpha}\left\{(6 a \alpha+3 b \alpha-6 b) T^{2}+\left(2 a \alpha^{2}+3 b \alpha^{2}-2 b \alpha\right) T^{3}\right.\right. \\
& \left.-r(2 a \alpha+b \alpha-2 b) T^{3}\right\}-P\left\{a r M^{2}+\frac{b r M^{3}}{2}\right. \\
& +\frac{a(a-1) r^{2} M^{3}}{6}-\frac{r k(2 a \alpha+b \alpha-2 b) M^{3}}{4 \alpha} \\
& -\frac{k}{2 \alpha}(2 a \alpha+b \alpha-2 b) M^{2} I_{C} T-\frac{k(a \alpha+b \alpha-b)}{2} T^{2} M \\
& -\frac{k\left(r-M r I_{C}-I_{C} M\right)}{4}(2 a \alpha+b \alpha-2 b) T M^{2} \\
& +\frac{k r\left(3-2 M I_{C}\right)(2 a \alpha+b \alpha-2 b)}{4 \alpha} T M^{2} \\
& \left.+\frac{k r\left(2-M I_{C}\right)(a \alpha+b \alpha-b)}{4} T^{2} M^{2}\right\} \\
& +\frac{C\left(1-M I_{C}\right)(2 a \alpha+b \alpha-2 b)}{2 \alpha} T \\
& +\frac{C\left(1-M I_{C}\right)(a \alpha+b \alpha-b)}{2} T^{2} \\
& +P\left\{M I_{C}-\left(I_{C}+r M I_{C}-r\right)-\frac{\left(r^{2}-2 r I_{C}-M r^{2} I_{C}\right)}{2} T^{2}\right\} \\
& \times\left(a M+\frac{b M^{2}}{2}+\frac{a r M^{2}}{2}+\frac{b r M^{3}}{6}+\frac{a^{2} r^{2} M^{3}}{6}\right. \\
& -\frac{k(2 a \alpha+b \alpha-2 b) M^{2}}{4 \alpha}+\frac{k(a \alpha-b) M^{3}}{6} \\
& \left.-\frac{k(2 a \alpha+b \alpha-2 b) M^{3}}{12 \alpha}\right)+\frac{P k r\left(1-M I_{C}\right)(2 a \alpha+b \alpha-2 b)}{4} T M^{3} \\
& +\frac{C\left(I_{C}+r M I_{C}-r\right)(2 a \alpha+b \alpha-2 b)}{2 \alpha} T^{2} \\
& +\left\{\frac{C\left(I_{C}+r M I_{C}-r\right)(a \alpha+b \alpha-b)}{2}+\frac{C\left(r^{2}-2 r I_{C}-M r^{2} I_{C}\right)(2 a \alpha+b \alpha-2 b)}{4 \alpha}\right\} T^{3} \\
& -\frac{P k\left(I_{C}+r M I_{C}-r\right)(2 a \alpha+b \alpha-2 b)}{2 \alpha} T^{2} M-\frac{P k\left(I_{C}-r\right)(2 a \alpha+b \alpha-2 b)}{2 \alpha} T^{2} M^{2} \\
& \left.-\frac{P k\left(r^{2}-2 r I_{C}\right)(2 a \alpha+b \alpha-2 b)}{4 \alpha} T^{3} M\right],
\end{aligned}
$$

Since there are $m$ cycles in the planning horizon $L$ then the present value of retailer's total cost over the planning horizon $L$ is

$$
\begin{gathered}
T_{C H}(T)=\sum_{n=0}^{m-1} T C_{2}(T) \mathrm{e}^{-r n T} \\
T C H_{2}(T)=\frac{\left(1-\mathrm{e}^{-r L}\right)}{\left(1-\mathrm{e}^{-r T}\right)} T_{C H}(T)
\end{gathered}
$$




$$
\begin{aligned}
& \mathrm{TCH}_{2}(T)=L\left[\frac{A r}{2}+P\left(r-I_{C}\right)\left\{a M+\frac{b M^{2}}{2}+\frac{a r M^{2}}{2}+\frac{b r M^{3}}{6}+\frac{a^{2} r^{2} M^{3}}{6}-\frac{k(2 a \alpha+b \alpha-2 b) M^{2}}{4 \alpha}\right.\right. \\
& \left.+\frac{k(a \alpha-b) M^{3}}{6}-\frac{k r(2 a \alpha+b \alpha-2 b) M^{3}}{12 \alpha}\right\}+\frac{\operatorname{PrMI}_{C}}{2}\left\{a M+\frac{b M^{2}}{2}+\frac{a r M^{2}}{2}\right. \\
& \left.-\frac{k M^{2}(2 a \alpha+b \alpha-2 b)}{4 \alpha}\right\}+\left\{\frac{P k M^{2} I_{C}}{2 \alpha}+\frac{P k M^{2}\left(r-M r I_{C}-M I_{C}\right)}{4}-\frac{P k r M^{3}\left(3-2 M I_{C}\right)}{4 \alpha}\right. \\
& \left.+\left(\frac{P k r M^{3}}{4}+\frac{C}{2 \alpha}-\frac{C M I_{C}}{2 \alpha}\right)\right\}(2 a \alpha+b \alpha-2 b)+\left[\frac{A r^{2}}{4}-\frac{P a r^{3} M^{2}}{4}-\frac{P b r^{3} M^{3}}{8}-\frac{P a(a-1) r^{4} M^{3}}{6}\right. \\
& +\left\{\frac{h_{C}}{4 \alpha}+\frac{C\left(I_{C}+M r I_{C}-r\right)}{2 \alpha}-\frac{P k M\left(I_{C}+M r I_{C}-r\right)}{2 \alpha}-\frac{P k M^{2}\left(I_{C}+M r I_{C}-r\right)}{2 \alpha}\right. \\
& +\frac{P k r M^{3}\left(I_{C}-r\right)}{4}+\frac{P k r M^{2} I_{C}}{4 \alpha}+\frac{P k r M^{2}\left(r-M r I_{C}-M I_{C}\right)}{8}-\frac{P k r^{2} M^{2}\left(3-2 M I_{C}\right)}{8 \alpha} \\
& \left.+\frac{C r\left(1-M I_{C}\right)}{4 \alpha}+\frac{P k r^{2} M^{3}}{8}+\frac{P k r^{3} M^{3}}{16 \alpha}\right\}(2 a \alpha+b \alpha-2 b)+\left\{\frac{P k M}{2}+\frac{C\left(1-M I_{C}\right)}{2}\right. \\
& \left.-\frac{P k r M^{2}\left(1-M I_{C}\right)}{4}+\frac{P k r^{2} M^{3}}{4}\right\}(a \alpha+b \alpha-b)+\frac{P}{4}\left\{M r^{2} I_{C}-2\left(2 r^{2}-r I_{C}\right)\left(a M+\frac{b M^{2}}{2}\right.\right. \\
& \left.+\frac{a r M^{2}}{2}+\frac{b r M^{3}}{6}+\frac{a^{2} r^{2} M^{3}}{6}-\frac{k M^{2}(2 a \alpha+b \alpha-2 b)}{4 \alpha}+\frac{k(a \alpha-b) M^{3}}{6}-\frac{k r M^{3}(2 a \alpha+b \alpha-2 b)}{12 \alpha}\right) \\
& \left.\left.+2 M r^{2} I_{C}\left(a M+\frac{b M^{2}}{2}+\frac{a r M^{2}}{2}-\frac{k M^{2}(2 a \alpha+b \alpha-2 b)}{4 \alpha}\right)\right\}\right]+\left[\frac{h_{C}(2 a \alpha+3 b \alpha-2 b)}{12}\right. \\
& +\left\{\frac{C\left(r^{2}-2 r I_{C}-M r^{2} I_{C}\right)}{4 \alpha}-\frac{P k r M^{2}\left(r^{2}-2 r I_{C}\right)}{4 \alpha}+\frac{P k r M^{2}\left(r^{2}-2 r I_{C}\right)}{4}+\frac{P k M^{2}\left(r^{2}-2 r I_{C}\right)}{4}\right. \\
& -\frac{P k M\left(r^{2}-2 r I_{C}-M r^{2} I_{C}\right)}{4}+\left(\frac{C r}{4}-\frac{P k r M}{4 \alpha}\right)\left(I_{C}+M r I_{C}-r\right)+\frac{C r^{2}\left(1-M I_{C}\right)}{8 \alpha}+\frac{r h_{C}}{24 \alpha} \\
& \left.+\frac{P k r^{2} M^{2} I_{C}}{8 \alpha}+\frac{P k r^{3} M^{2}}{16}\right\}(2 a \alpha+b \alpha-2 b)+\left\{\frac{C\left(I_{C}+r M I_{C}-r\right)}{2}-\frac{P k r M^{2}\left(I_{C}-r\right)}{4}\right. \\
& \left.+\frac{C r\left(1-M I_{C}\right)}{4}-\frac{P k r^{2} M^{2}}{4}+\frac{P k r M}{4}\right\}(a \alpha+b \alpha-b)+\frac{P r^{2} I_{C}}{4}\left(a M+\frac{b M^{2}}{2}+\frac{a r M^{2}}{2}\right. \\
& \left.\left.-\frac{k M^{2}(2 a \alpha+b \alpha-2 b)}{4 \alpha}\right)\right] T^{2}+\left[\left\{\frac{r h_{C}}{24}+\frac{r^{2} h_{C}}{48 \alpha}+\frac{C r\left(r^{2}-2 r I_{C}-M r^{2} I_{C}\right)}{8 \alpha}-\frac{P k r M\left(r^{2}-2 r I_{C}\right)}{8 \alpha}\right.\right. \\
& \left.+\frac{C\left(I_{C}+r M I_{C}-r\right)}{2 \alpha}-\frac{P k r^{2} M\left(I_{C}-r\right)}{8 \alpha}\right\}(2 a \alpha+b \alpha-2 b)+\left\{\frac{C r\left(I_{C}+r M I_{C}-r\right)}{4}\right. \\
& \left.\left.+\left(\frac{C r^{2}}{8}+\frac{P k r^{4}}{16}\right)\left(1-M I_{C}\right)+\frac{P k r^{2} M}{8}\right\}(a \alpha+b \alpha-b)-\frac{P a r^{2} M\left(r^{2}-2 r I_{C}\right)}{8}\right] T^{3} \\
& +\frac{1}{T}\left[A-\frac{P(r+2)}{2}\left\{a r M^{2}+\frac{b r M^{3}}{2}+\frac{a(a-1)^{2} M^{3}}{6}-\frac{k r M^{3}(2 a \alpha+b \alpha-2 b)}{4 \alpha}\right\}\right. \\
& \left.\left.+P M I_{C}\left(a M+\frac{b M^{2}}{2}+\frac{a r M^{2}}{2}-\frac{k M^{2}(2 a \alpha+b \alpha-2 b)}{4 \alpha}\right)\right]\right]
\end{aligned}
$$


The necessary condition for $\mathrm{TCH}_{2}(T)$ to be minimum is that $\frac{\mathrm{d} T C H_{2}(T)}{\mathrm{d} T}=0$ and the sufficient condition is $\frac{\mathrm{d}^{2} T C H_{2}(T)}{\mathrm{d} T^{2}} \succ 0$ at the optimum value of $T$.

$$
\begin{aligned}
& \frac{\mathrm{d} T C H_{2}(T)}{\mathrm{d} T}=L\left[\frac{A r^{2}}{4}-\frac{P a r^{3} M^{2}}{4}-\frac{P b r^{3} M^{3}}{8}-\frac{P a(a-1) r^{4} M^{3}}{6}+\left\{\frac{h_{C}}{4 \alpha}+\frac{C}{2 \alpha}\left(I_{C}+M r I_{C}-r\right)\right.\right. \\
& -\frac{P k M\left(I_{C}+M r I_{C}-r\right)}{2 \alpha}-\frac{P k M^{2}\left(I_{C}+M r I_{C}-r\right)}{2 \alpha}+\frac{P k r M^{3}\left(I_{C}-r\right)}{4}+\frac{P k r M^{2} I_{C}}{4 \alpha} \\
& +\frac{P k r M^{2}\left(r-M r I_{C}-M I_{C}\right)}{8}-\frac{P k r^{2} M^{2}\left(3-2 M I_{C}\right)}{8 \alpha}+\frac{C r\left(1-M I_{C}\right)}{4 \alpha}+\frac{P k r^{2} M^{3}}{8} \\
& \left.+\frac{P k r^{3} M^{3}}{16 \alpha}\right\}(2 a \alpha+b \alpha-2 b)+\left\{\frac{P k M}{2}+\frac{C\left(1-M I_{C}\right)}{2}-\frac{P k r M^{2}\left(1-M I_{C}\right)}{4}\right. \\
& \left.+\frac{P k r^{2} M^{3}}{4}\right\}(a \alpha+b \alpha-b)+\frac{P}{4}\left\{M r^{2} I_{C}-\left(2 r^{2}-r I_{C}\right)\left(a M+\frac{b M^{2}}{2}+\frac{a r M^{2}}{2}+\frac{b r M^{3}}{6}\right.\right. \\
& \left.+\frac{a^{2} r^{2} M^{3}}{6}-\frac{k M^{2}(2 a \alpha+b \alpha-2 b)}{4 \alpha}+\frac{k(a \alpha-b) M^{3}}{6}-\frac{k r M^{3}(2 a \alpha+b \alpha-2 b)}{12 \alpha}\right) \\
& \left.+2 M r^{2} I_{C}\left(a M+\frac{b M^{2}}{2}+\frac{a r M^{2}}{2}-\frac{k M^{2}(2 a \alpha+b \alpha-2 b)}{4 \alpha}\right)\right\}+2\left[\frac{h_{C}(2 a \alpha+3 b \alpha-2 b)}{12}\right. \\
& +\left\{\frac{C\left(r^{2}-2 r I_{C}-M r^{2} I_{C}\right)}{4 \alpha}-\frac{P k r M^{2}\left(r^{2}-2 r I_{C}\right)}{4 \alpha}+\frac{P k r M^{2}\left(r^{2}-2 r I_{C}\right)}{4}+\frac{P k M^{2}\left(r^{2}-2 r I_{C}\right)}{4}\right. \\
& -\frac{P k M\left(r^{2}-2 r I_{C}-M r^{2} I_{C}\right)}{4}+\left(\frac{C r}{4}-\frac{P k R M}{4 \alpha}\right)\left(I_{C}+M r I_{C}-r\right)+\frac{C r^{2}\left(1-M I_{C}\right)}{8 \alpha}+\frac{r h_{C}}{24 \alpha} \\
& \left.+\frac{P k r^{2} M^{2} I_{C}}{8 \alpha}+\frac{P k r^{3} M^{2}}{16}\right\}(2 a \alpha+b \alpha-2 b)+\left\{\frac{C\left(I_{C}+r M I_{C}-r\right)}{2}-\frac{P k r M^{2}\left(I_{C}-r\right)}{4}\right. \\
& \left.+\frac{C r\left(1-M I_{C}\right)}{4}-\frac{P k r^{2} M^{2}}{4}+\frac{P k r M}{4}\right\}(a \alpha+b \alpha-b)+\frac{P r^{2} I_{C}}{4}\left(a M+\frac{b M^{2}}{2}+\frac{a r M^{2}}{2}\right. \\
& \left.\left.-\frac{k M^{2}(2 a \alpha+b \alpha-2 b)}{4 \alpha}\right)\right] T+\left[\left\{\frac{r h_{C}}{24}+\frac{r^{2} h_{C}}{48 \alpha}+\frac{C r\left(r^{2}-2 r I_{C}-M r^{2} I_{C}\right)}{8 \alpha}-\frac{P k r M\left(r^{2}-2 r I_{C}\right)}{8 \alpha}\right.\right. \\
& \left.+\frac{C\left(I_{C}+r M I_{C}-r\right)}{2 \alpha}-\frac{P k r^{2} M\left(I_{C}-r\right)}{8 \alpha}\right\}(2 a \alpha+b \alpha-2 b)+\left\{\frac{C r\left(I_{C}+r M I_{C}-r\right)}{4}\right. \\
& \left.\left.+\left(\frac{C r^{2}}{8}+\frac{P k r^{4}}{16}\right)\left(1-M I_{C}\right)+\frac{P k r^{2} M}{8}\right\}(a \alpha+b \alpha-b)-\frac{P a r^{2} M\left(r^{2}-2 r I_{C}\right)}{8}\right] T^{2} \\
& -\frac{1}{T^{2}}\left[A-\frac{P(r+2)}{2}\left\{a r M^{2}+\frac{b r M^{3}}{2}+\frac{a(a-1)^{2} M^{3}}{6}-\frac{k r M^{3}(2 a \alpha+b \alpha-2 b)}{4 \alpha}\right\}\right. \\
& \left.\left.+P M I_{C}\left(a M+\frac{b M^{2}}{2}+\frac{a r M^{2}}{2}-\frac{k M^{2}(2 a \alpha+b \alpha-2 b)}{4 \alpha}\right)\right]\right] \text {, }
\end{aligned}
$$




$$
\begin{aligned}
\frac{\mathrm{d}^{2} T C H_{2}(T)}{\mathrm{d} T^{2}}= & L\left[\frac{h_{C}(2 a \alpha+3 b \alpha-2 b)}{12}+\left\{\frac{C\left(r^{2}-2 r I_{C}-M r^{2} I_{C}\right)}{4 \alpha}-\frac{P k r M^{2}\left(r^{2}-2 r I_{C}\right)}{4 \alpha}\right.\right. \\
& +\frac{P k r M^{2}\left(r^{2}-2 r I_{C}\right)}{4}+\frac{P k M^{2}\left(r^{2}-2 r I_{C}\right)}{4}-\frac{P k M\left(r^{2}-2 r I_{C}-M r^{2} I_{C}\right)}{4} \\
& +\left(\frac{C r}{4}-\frac{P k R M}{4 \alpha}\right)\left(I_{C}+r M I_{C}-r\right)+\frac{C r^{2}\left(1-M I_{C}\right)}{8 \alpha}+\frac{r h_{C}}{24 \alpha}+\frac{P k r^{2} M^{2} I_{C}}{8 \alpha} \\
& \left.+\frac{P k r^{3} M^{2}}{16}\right\}(2 a \alpha+b \alpha-2 b)+\left\{\frac{C\left(I_{C}+r M I_{C}-r\right)}{2}-\frac{P k r M^{2}\left(I_{C}-r\right)}{4}\right. \\
& \left.+\frac{C r\left(1-M I_{C}\right)}{4}-\frac{P k r^{2} M^{2}}{4}+\frac{P k r M}{4}\right\}(a \alpha+b \alpha-b)+\frac{P r^{2} I_{C}}{4}\left(a M+\frac{b M^{2}}{2}\right. \\
& \left.+\frac{a r M^{2}}{2}-\frac{k M^{2}(2 a \alpha+b \alpha-2 b)}{4 \alpha}\right]+6\left[\left\{\frac{r h_{C}}{24}+\frac{r^{2} h_{C}}{48 \alpha}+\frac{C r\left(r^{2}-2 r I_{C}-M r^{2} I_{C}\right)}{8 \alpha}\right.\right. \\
& \left.-\frac{P k r M\left(r^{2}-2 r I_{C}\right)}{8 \alpha}+\frac{C\left(I_{C}+r M I_{C}-r\right)}{2 \alpha}-\frac{P k r^{2} M\left(I_{C}-r\right)}{8 \alpha}\right\}(2 a \alpha+b \alpha-2 b) \\
& +\left\{\frac{C r\left(I_{C}+r M I_{C}-r\right)}{4}+\left(\frac{C r^{2}}{8}+\frac{P k r^{4}}{16}\right)\left(1-M I_{C}\right)+\frac{P k r^{2} M}{8}\right\}(a \alpha+b \alpha-b) \\
& \left.\left.-\frac{k r M^{3}(2 a \alpha+b \alpha-2 b)}{4 \alpha}\right\}+P M I_{C}\left(a M+\frac{b M^{2}}{2}+\frac{a r M^{2}}{2}-\frac{k M^{2}(2 a \alpha+b \alpha-2 b)}{4 \alpha}\right)\right] \\
& \left.-\frac{P r^{2} M\left(r^{2}-2 r I_{C}\right)}{8}\right] T+\frac{2}{T^{3}}\left[A-\frac{P(r+2)}{2}\left\{a r M^{2}+\frac{b r M^{3}}{2}+\frac{a(a-1)^{2} M^{3}}{6}\right.\right. \\
& \\
&
\end{aligned}
$$

\section{Numerical Parameters}

Let us consider the following parameters in the appropriate units

$$
a=100, b=3, k=10, C=6, \alpha=12, A=4, h_{C}=5, s_{C}=8, P=20, r=1.20, M=2, I_{C}=0.8, L=50
$$

\subsection{Numerical Example I}

When $M \geq T$ then solving the equation $\frac{\mathrm{d} T C H_{1}}{\mathrm{~d} T}=0$, we find the optimum value of $T$ satisfying the condition $\frac{\mathrm{d}^{2} T C H_{1}}{\mathrm{~d} T^{2}} \succ 0$.

Since $\frac{\mathrm{d}^{2} T C H_{1}}{\mathrm{~d} T^{2}}=2.11046 \times 10^{7}$

As we increase the parameter $r$ then the value of total cost decreases.

As we increase the parameter $M$ then the value of total cost decreases.

\subsection{Numerical Parameters}

Let us consider the following parameters in the appropriate units

$$
a=100, b=3, k=10, C=6, \alpha=12, A=4, h_{C}=5, s_{C}=8, P=20, r=0.05, M=2, I_{C}=0.8, L=50
$$




\subsection{Numerical Example II}

When $M \prec T$ then solving the equation $\frac{\mathrm{d} T C H_{2}}{\mathrm{~d} T}=0$, we find the optimum value of $T$ satisfying the condition $\frac{\mathrm{d}^{2} T C \mathrm{H}_{2}}{\mathrm{~d} T^{2}} \succ 0$. Since $\frac{\mathrm{d}^{2} T C \mathrm{H}_{2}}{\mathrm{~d} T^{2}}=-146586 \times 10^{9}$ so the total cost is maximum.

As we increase the parameter $r$ then the value of total cost increases.

As we increase the parameter $M$ then the value of total cost decreases.

\section{Conclusion}

In this paper, we proposed an inventory model for perishable items with time varying stock dependent demand under inflation and time discounting. In the numerical analysis we study the effect of the change of the parameters $r$ and $M$ on the optimal solution. From Table 1 and Table 2 we observe that as we increase the parameters $r$ and $M$ then the replenishment cycle length increases and the corresponding total cost decreases since the total cost decreases and the revenue increases on his sell to pay the purchasing cost and in the case when $M \geq T$ no interest will be charged by the supplier from the retailer. When the inflation rate increases then the retailer wants to short the length of replenishment cycle. From Table 3 we see that as we increase the parameter $r$ then the value of replenishment cycle length and total cost decreases. From Table 4 we see that as we increase the parameter $M$ then the value of replenishment cycle length increases and the value of total cost decreases. Thus we see that when the credit period is short then the retailer wants to order less and decrease the chargeable interest. When the credit period is large enough then the retailer wants to order more and he earns enough revenue on his sell to pay the purchasing cost therefore the credit period attracts the retailer to buy more or less.

\section{References}

[1] Buzacott, J.A. (1975) Economic Order Quantity Model under Inflation. Operational Research Quarterly, 26, 553-558. http://dx.doi.org/10.2307/3008214

[2] Silver, E.A. and Peterson, R. (1985) Decision Systems for Inventory Management and Production Planning. 2nd Edition, John Wiley \& Sons, New York.

[3] Baker, R.C. and Urban, T.L. (1988) A Deterministic Inventory System with an Inventory Level Dependent Demand Rate. Journal of Operational Research Society, 39, 823-831. http://dx.doi.org/10.1057/jors.1988.142

[4] Mandal, B.N. and Phaujdar, S. (1989) An Inventory Model for Deteriorating Items and Stock Dependent Consumption Rate. Journal of Operational Research Society, 40, 483-488. http://dx.doi.org/10.1057/jors.1989.75

[5] Vrat, P. and Padmanabhan, G. (1990) An Inventory Model with Stock Dependent Consumption Rate under Inflation. Engineering Costs and Production Economics, 19, 379-383. http://dx.doi.org/10.1016/0167-188X(90)90068-S

[6] Padmanabhan, G. and Vrat, P. (1995) EOQ Models for Perishable Items with Stock Dependent Selling Rate. European Journal of Operational Research, 86, 281-292. http://dx.doi.org/10.1016/0377-2217(94)00103-J

[7] Bose, S., Goswami, A. and Choudhuri, K.S. (1995) An EOQ Model for Deteriorating Items with Linear Time Dependent Demand Rate and Shortages under Inflation and Time Discounting. Journal of Operational Research Society, 46, 771-782. http://dx.doi.org/10.1057/jors.1995.107

[8] Mandal, M. and Maiti, M. (1999) An Inventory Model of Damageable Products Having Some Units in Hand with Stock Dependent Demand, Variable Replenishment Rate. Applied Mathematical Modeling, 23, 799-807. http://dx.doi.org/10.1016/S0307-904X(99)00018-9

[9] Chung, K.-J. and Lin, C.-N. (2001) An Optimal Replenishment Policy for an Inventory Model for Deteriorating Items with Time Discounting. Computers and Operations Research, 28, 67-83. http://dx.doi.org/10.1016/S0305-0548(99)00087-8

[10] Chang, C.T. (2004) An EOQ Model for Deteriorating Items under Inflation When Supplier Credits Linked to Order Quantity. International Journal of Production Economics, 88, 307-316.

[11] Dye, C.Y. and Ouyang, L.Y. (2005) An EOQ Model for Perishable Items with Stock Dependent Selling Rate by Allowing Shortages,. European Journal of Operational Research, 163, 776-783. http://dx.doi.org/10.1016/j.ejor.2003.09.027

[12] Hou, K.L. (2006) An Inventory Model for Deteriorating Items with Stock Dependent Consumption Rate and Shortages under Inflation and Time Discounting. European Journal of Operational Research, 168, 463-474. 
http://dx.doi.org/10.1016/j.ejor.2004.05.011

[13] Jaggi, C.K., Aggarwal, K.K. and Goel, S.K. (2006) An Optimal Ordering Policy for an Inventory Model of Deteriorating Items under Inflation Induced Demand. International Journal of Production Economics, 103, 707-714. http://dx.doi.org/10.1016/j.ijpe.2006.01.004

[14] Sana, S.S. and Chaudhuri, K.S. (2008) A Deterministic EOQ Model for Deteriorating Items with Delay in Payments Offered by the Supplier. European Journal of Operational Research, 184, 509-533. http://dx.doi.org/10.1016/j.ejor.2006.11.023

[15] Valliathal, M. and Uthayakumar, R. (2009) An EOQ Model for Perishable Items with Stock and Time Dependent Selling Rate by Allowing Shortages. APRN Journal of Engineering and Applied Sciences, 4, 8-13.

[16] Roy, A., Maiti, M.K., Kar, S. and Maiti, M. (2009) An Inventory Model for Deteriorating Items with Stock Dependent Demand under Fuzzy Inflation and Time Discounting over a Random Planning Horizon. Applied Mathematical Modeling, 33, 744-759. http://dx.doi.org/10.1016/j.apm.2007.12.015

[17] Sana, S.S. (2010) An Inventory Model for Time Varying Deteriorating Items with Optimal Selling Price and Lot Size by Allowing Shortages. Applied Mathematics and Computation, 217, 185-194. http://dx.doi.org/10.1016/j.amc.2010.05.040

[18] Chang, C.T., Teng, J.T. and Goyal, S.K. (2010) Optimal Replenishment Policies for Non Instantaneous Deteriorating Items with Stock Dependent Demand. International Journal of Production Economics, 123, 62-68. http://dx.doi.org/10.1016/j.ijpe.2009.06.042

[19] Sarkar, B., Sana, S.S. and Chaudhuri, K. (2011) An EMQ Model for an Imperfect Production Process for Time Varying Demand under Inflation and Time Value of Money. Expert Systems with Applications, 38, 13543-13548.

[20] Yan, X. (2012) An EOQ Model for Perishable Items with Freshness Dependent Demand and Partial Backlogging. International Journal of Control and Automation, 5, 19-38.

[21] Nagare, M. and Dutta, P. (2012) A Continuous Review Inventory Model for Perishable Products with Inventory Dependent Demand. Proceeding of the International Multi Conference of Engineers and Computer Scientists Hong-Kong, 14-16 March 2012, 1513-1517.

[22] Sana, S.S. (2013) A Sales Team’s Initiatives and Stock Sensitive Demand under Production Control Policy. Economic Modeling, 31, 783-788. http://dx.doi.org/10.1016/j.econmod.2013.01.026

[23] Yang, S., Lee, C. and Zhang, A.M. (2013) An Inventory Model for Perishable Products with Stock Dependent Demand and Trade Credit under Inflation. Mathematical Problems in Engineering, 2013, 1-10. http://dx.doi.org/10.1155/2013/702939 